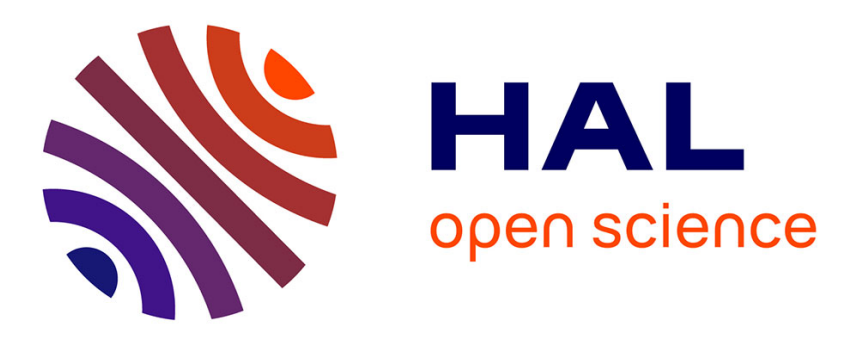

\title{
Crescent-Shaped Electron Distributions at the Nonreconnecting Magnetopause: Magnetospheric Multiscale Observations
}

B.-B Tang, W. Li, D B Graham, A C Rager, C. Wang, Yu V Khotyaintsev, B. Lavraud, H. Hasegawa, Y.-C Zhang, L Dai, et al.

\section{To cite this version:}

B.-B Tang, W. Li, D B Graham, A C Rager, C. Wang, et al.. Crescent-Shaped Electron Distributions at the Nonreconnecting Magnetopause: Magnetospheric Multiscale Observations. Journal of Geophysical Research Space Physics, 2019, 10.1029/2019GL082231 . hal-02372809

\section{HAL Id: hal-02372809 https://hal.science/hal-02372809}

Submitted on 20 Nov 2019

HAL is a multi-disciplinary open access archive for the deposit and dissemination of scientific research documents, whether they are published or not. The documents may come from teaching and research institutions in France or abroad, or from public or private research centers.
L'archive ouverte pluridisciplinaire HAL, est destinée au dépôt et à la diffusion de documents scientifiques de niveau recherche, publiés ou non, émanant des établissements d'enseignement et de recherche français ou étrangers, des laboratoires publics ou privés. 


\section{Geophysical Research Letters}

\author{
RESEARCH LETTER \\ 10.1029/2019GL082231 \\ Key Points: \\ - Agyrotropic electron crescents are \\ found in a nonreconnecting current \\ sheet at the flank magnetopause \\ - These electron distributions are \\ generated by finite gyroradius effect \\ after magnetic curvature scattering \\ - The observed electron crescents can \\ excite upper hybrid waves
}

Supporting Information:

- Supporting Information S1

- Figure S1

Correspondence to:

C. Wang,

cwang@spaceweather.ac.cn

Citation:

Tang, B.-B., Li, W. Y., Graham, D. B., Rager, A. C., Wang, C.,

Khotyaintsev, Y. V., et al. (2019).

Crescent-shaped electron

distributions at the nonreconnecting

magnetopause: Magnetospheric

multiscale observations.

Geophysical Research

Letters, 46, 3024-3032.

https://doi.org/10.1029/2019GL082231

Received 31 JAN 2019

Accepted 26 FEB 2019

Accepted article online 1 MAR 2019

Published online 19 MAR 2019

(C)2019. American Geophysical Union. All Rights Reserved.

\section{Crescent-Shaped Electron Distributions at the Nonreconnecting Magnetopause: Magnetospheric Multiscale Observations}

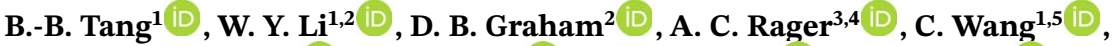 \\ Yu. V. Khotyaintsev ${ }^{(i)}$, B. Lavraud ${ }^{(D)}$, H. Hasegawa ${ }^{7}$, Y.-C. Zhang' ${ }^{(D)}$, L. Dai ${ }^{1}$ (i), \\ B. L. Giles ${ }^{4}$ (D) J. C. Dorelli ${ }^{4}$, C. T. Russell ${ }^{8}$ iD, P.-A. Lindqvist ${ }^{9}$ iD, R. E. Ergun ${ }^{10}$ iD, \\ and J. L. Burch ${ }^{11}$ iD
}

${ }^{1}$ State Key Laboratory of Space Weather, National Space Science Center, Chinese Academy of Sciences, Beijing, China,
${ }^{2}$ Swedish Institute of Space Physics, Uppsala, Sweden, ${ }^{3}$ Department of Physics, Catholic University of America,
Washington, DC, USA, ${ }^{4}$ NASA Goddard Space Flight Center, Greenbelt, MD, USA, ${ }^{5}$ College of Earth and Planetary
Sciences, University of Chinese Academy of Sciences, Beijing, China, ${ }^{6}$ Institut de Recherche en Astrophysique et
Planetologie, Universite de Toulouse, Toulouse, France, ${ }^{7}$ Institute of Space and Astronautical Science, Japan Aerospace
Exploration Agency, Sagamihara, Japan, ${ }^{8}$ Department of Earth and Space Sciences, University of California, Los
Angeles, CA, USA, ${ }^{9} \mathrm{KTH}$ Royal Institute of Technology, Stockholm, Sweden, ${ }^{10}$ Laboratory of Atmospheric and Space
Physics, University of Colorado Boulder, Boulder, CO, USA, ${ }^{11}$ Southwest Research Institute, San Antonio, TX, USA

Abstract Crescent-shaped electron distributions perpendicular to the magnetic field are an important indicator of the electron diffusion region in magnetic reconnection. They can be formed by the electron finite gyroradius effect at plasma boundaries or by demagnetized electron motion. In this study, we present Magnetospheric Multiscale mission observations of electron crescents at the flank magnetopause on 20 September 2017, where reconnection signatures are not observed. These agyrotropic electron distributions are generated by electron gyromotion at the thin electron-scale magnetic boundaries of a magnetic minimum after magnetic curvature scattering. The variation of their angular range in the perpendicular plane is in good agreement with predictions. Upper hybrid waves are observed to accompany the electron crescents at all four Magnetospheric Multiscale spacecraft as a result of the beam-plasma instability associated with these agyrotropic electron distributions. This study suggests electron crescents can be more frequently formed at the magnetopause.

Plain Language Summary In this study, we present Magnetospheric Multiscale mission observations of electron crescents at the flank magnetopause and these agyrotropic electron distributions are formed at thin electron-scale magnetic boundaries after electron pitch angle scattering by the curved magnetic field. These results suggest that agyrotropic electron distributions can be more frequently formed at the magnetopause: (1) magnetic reconnection is not necessary, although electron crescents are taken as one of the observational signatures of the electron diffusion region, and (2) agyrotropic electron distributions can cover a large local time range to the flank magnetopause. In addition, upper hybrid waves accompanied with the electron crescents are observed as a result of the beam-plasma interaction associated with these agyrotropic electron distributions. This suggests that high-frequency waves play a role in electron dynamics through wave-particle interactions.

\section{Introduction}

Charged particles gyrate around magnetic field lines under the Lorentz force, typically forming gyrotropic distributions in the plane perpendicular to the magnetic field. These gyrotropic distributions may not survive either when a plasma/magnetic field boundary has a width comparable to the particle's gyroradius ( $\rho=\frac{m v_{\perp}}{q B}$ ) due to finite gyroradius effect or when the particles become decoupled from the magnetic field. The observations for ion's agyrotropic distributions have been widely reported (e.g., Graham et al., 2017a; Phan et al., 2016; Wang et al., 2016, and reference therein). On the other hand, due to electron's small mass, such agyrotropic distributions of electrons are difficult to investigate in space because in situ measurements 
in previous space missions are too slow to electron scales. However, the recently launched Magnetospheric Multiscale (MMS) mission (Burch et al., 2016), measuring charged particle orders of magnitude faster, provides a good opportunity to explore electron-scale physics (e.g., Burch \& Phan, 2016; Chen et al., 2016; Lavraud et al., 2016; Phan et al., 2018; Khotyaintsev et al., 2016).

One of the most active regions for electrons in space is the electron diffusion region (EDR) of magnetic reconnection, where magnetic energy converts to particle energy rapidly. In and near EDRs observed by MMS at the dayside magnetopause, crescent-shaped agyrotropic electron distributions perpendicular to the magnetic field have been observed at the magnetospheric side of the EDR (e.g., Burch et al., 2016; Chen et al., 2016; Webster et al., 2018) as a result of electron meandering motion in the thin magnetic field reversal (Lapenta et al., 2017), which are consistent with particle simulations (e.g., Bessho et al., 2017; Hesse et al., 2014; Shay et al., 2016). The crescent electrons have a cutoff energy, depending on the electric field in the normal direction and the penetration distance from the separatrix (Egedal et al., 2016), so that they are not filled in at lower energies. Electron crescents were also found at the magnetosheath side of the Hall region during an MMS near-EDR crossing (Norgren et al., 2016) owing to electron finite gyroradius effect. These electron crescents can become oblique due to the presence of the parallel electric field (Burch \& Phan, 2016) or the electron inertia if the magnetic field lines rotate rapidly (Egedal et al., 2018). Therefore, agyrotropic electron crescents, along with other signatures (such as intense current density and enhanced energy dissipation), are taken as direct observational indicators of an EDR or near-EDR event (e.g., Fuselier et al., 2017; Webster et al., 2018). These agyrotropic electron distributions (electron beams and/or crescents) can also behave as a source for electron diffusion and thermalization near the EDR through wave-particle interactions, as they are unstable to generate high-frequency plasma waves (e.g., upper hybrid, UH, or Langmuir waves; Burch et al., 2018; Graham et al., 2017b).

However, the generation of agyrotropic electron crescents by electron finite gyroradius effect is not necessarily related to the reconnection EDR, if electrons cross a thin electron-scale magnetic/plasma boundary through gyromotion. In this study, we report such a case at the flank magnetopause. Crescent-shaped agyrotropic electron distributions are formed at the thin electron-scale magnetic boundaries after electron pitch angle scattering by the curved magnetic field. UH waves accompanied with the electron crescents are identified. This observation suggests that the electron crescents can be more frequently formed at the magnetopause.

\section{Observations}

We investigate a magnetopause crossing of MMS between 23:41:40 and 23:42:20 UT on 20 September 2017. During this crossing, the four MMS spacecraft were located at the duskside flank magnetopause, and the position in the geocentric solar magnetospheric (GSM) coordinate is approximately [-10.9, 20.6, 1.3] Earth radii $\left(R_{E} ;\right.$ Figure 1a). The spacecraft were in a tetrahedron formation with an average separation of $\sim 20 \mathrm{~km}$ (Figure 1b). We use ion and electron data from the fast plasma investigation (Pollock et al., 2016), magnetic field data from the fluxgate magnetometer (Russell et al., 2014), and electric field data from electric field double probes (Ergun et al., 2014; Lindqvist et al., 2014). All data are presented in high-resolution burst mode, and the regular burst mode resolution for electrons is $30 \mathrm{~ms}$.

Figure 1 shows an overview of the outbound magnetopause crossing. The MMS spacecraft are initially located at the magnetospheric side, characterized by low plasma density (Figure 1d), weak ion velocity (Figure 1e), and an energetic magnetospheric ion population $(\sim 10 \mathrm{keV}$; Figure 1i). At the magnetosheath side, the plasma is much denser, colder, and moves tailward at a speed $\left(-V_{X}\right)$ of $\sim 240 \mathrm{~km} / \mathrm{s}$. Between 23:41:55 and 23:42:05 UT, the plasma parameters are in a transition state from the magnetosphere to the magnetosheath, which are bounded by two large magnetic field rotations (Figure 1c). Within the yellow-shaded time interval, MMS observes deep magnetic field depressions and large electron bulk flows (Figures 1c and $1 \mathrm{~g})$, which are the focus of this study.

A detailed plot is then presented in Figure 2, and the vectors have been transformed into the local boundary normal LMN coordinates based on minimum variance analysis of $\mathbf{B}$ between 23:41:53.00 and 23:41:56.50 UT. Here $\mathbf{L}=[-0.50,0.86,0.07]$ is the direction of the maximum variance of the magnetic field, $\mathbf{N}=[0.83,0.45$, $0.34]$ is the minimum variance direction, and $\mathbf{M}=[-0.25,-0.23,0.94]$ completes the third orthogonal axis. 

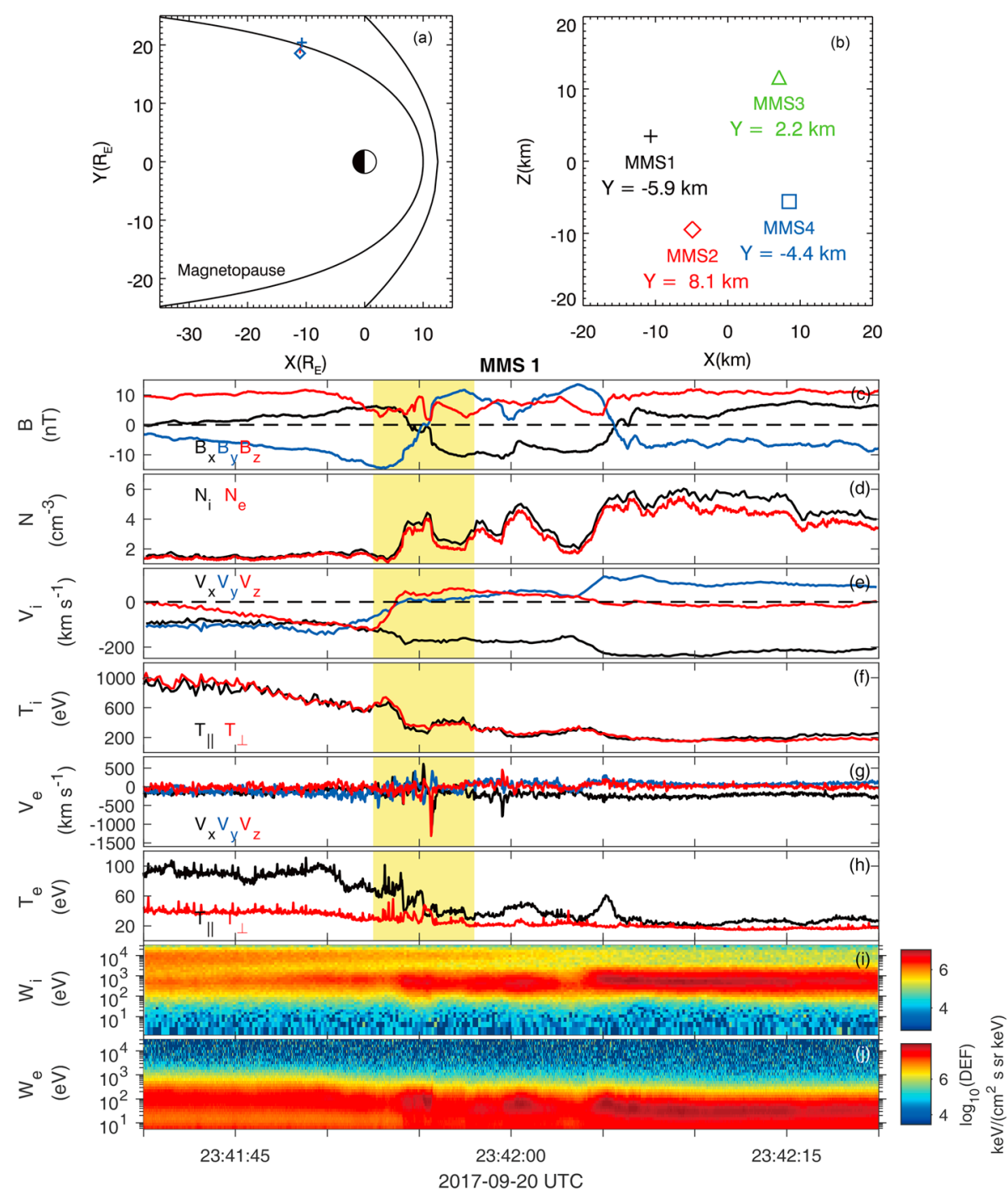

Figure 1. Overview of MMS crossings at the flank magnetopause. (a) Equatorial projection of MMS orbit in geocentric solar magnetospheric is from 20-09-2017/22:00 UT to 21-09-2017/02:00 UT, and the diamond marks the starting position. (b) MMS spacecraft relative positions. (c) Magnetic field. (d) Ion and electron number density. (e) Ion bulk velocity. (f) Ion parallel and perpendicular temperature. (g) Electron bulk velocity. (h) Electron parallel and perpendicular temperature. (i) Ion differential energy flux. (j) Electron differential energy flux. A detailed plot of the yellow-shaded time interval is presented in Figure 2. MMS = Magnetospheric Multiscale.

From four-spacecraft timing method of $B_{L}$, the boundary speed is estimated to be $\mathbf{V} \approx-85 \times[0.79,0.61$, $0.12] \mathrm{km} / \mathrm{s}(\mathrm{GSM})$, roughly aligned with $\mathbf{N}$. The thickness of the entire current sheet (between 23:41:54.1 and 23:31:56.0 UT) is thus about $160 \mathrm{~km}$, which is comparable to the ion thermal gyroradius $\left(\rho_{i} \approx 140 \mathrm{~km}\right)$. During the current sheet crossing, no fast ion jet is observed (Figure 2d). A clear magnetic field depression is detected in the central current sheet, and its minimum magnitude is about $1.9 \mathrm{nT}$ (Figure 2a). At the two edges of this depressed magnetic field region (marked by two vertical black lines in Figure 2), there are strong electric fields and large electron bulk flows (Figures $2 \mathrm{~b}$ and $2 \mathrm{e}$ ). The electrons follow $\mathbf{E} \times \mathbf{B}$ drift motion during the current sheet crossing (not shown). The current density is calculated from particle moments (Figure 2f) and reaches a maximum of $850 \mathrm{nA} / \mathrm{m}^{2}$, which is quantitatively consistent with the magnetic depressions if simply assuming a planar current sheets geometry at the two edges. The energy dissipation in the electron rest frame $\left(\mathbf{J} \cdot E^{\prime}=\mathbf{J} \cdot \mathbf{E}+V_{e} \times \mathbf{B}\right)$, presented in the satellite spin plane (Figure $2 \mathrm{~g}$ ), represents the rate of nonideal energy conversion from the magnetic field to the particles. The variation of $\mathbf{J} \cdot E^{\prime}$ is small, and its value is zero on average. 

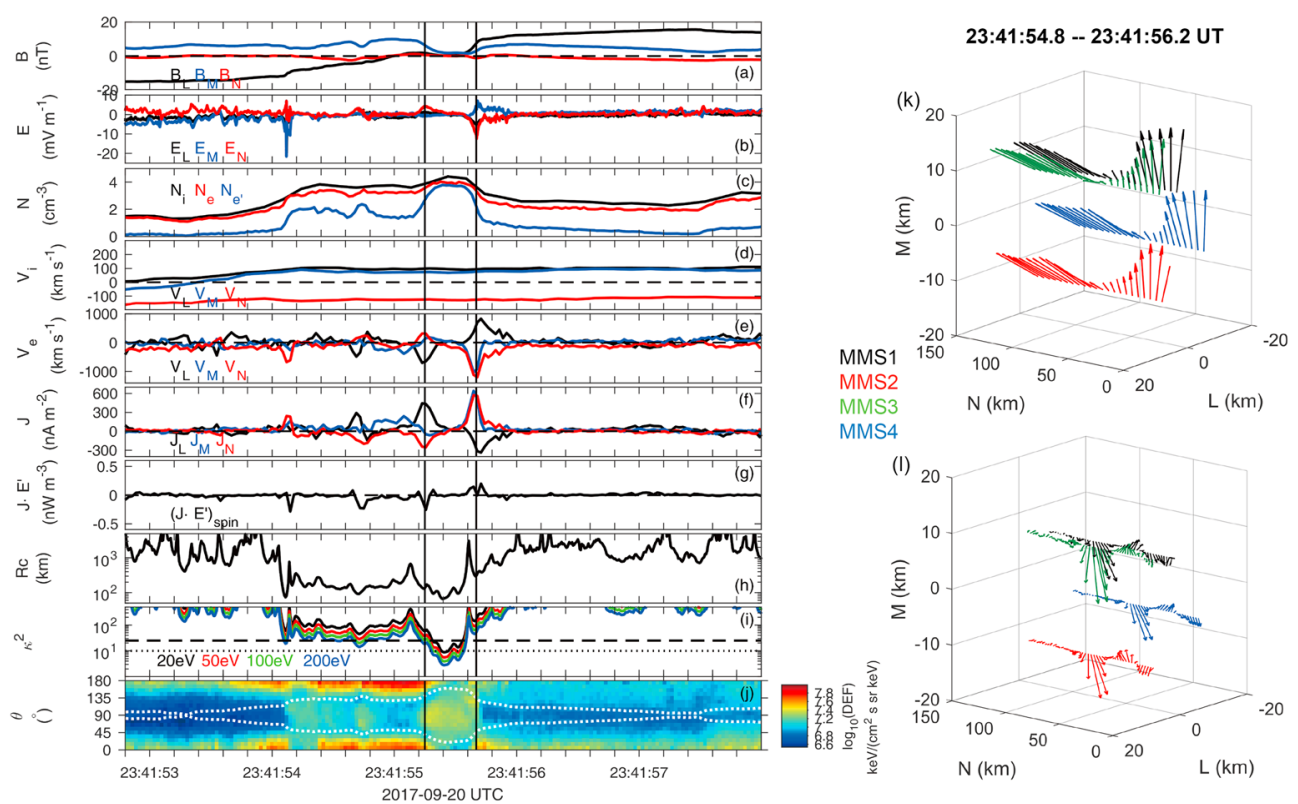

Figure 2. MMS observations around the magnetic minima in LMN coordinate. The left column: (a) Magnetic field. (b) Electric field. (c) Number density of ion and electron, and the partial electron density with pitch angles between two dashed lines in panel (i). (d) Ion velocity. (e) Electron velocity. (f) Electric current calculated from FPI particle moments. (g) Energy dissipation $\left(\mathbf{J} \cdot E^{\prime}\right)$ in the satellite spin plane, $(\mathrm{h})$ Magnetic curvature radius $\left(R_{c}\right)$. (i) Adiabatic parameter defined by $\kappa^{2}=R_{c} / \rho_{e}$. (j) Electron pitch angle distributions of all energies. The two vertical black lines mark the peak of the current densities near the magnetic minima. The right column: 3-D views of (k) magnetic field and (l) electron velocity vectors. MMS = Magnetospheric Multiscale.

Since the magnetic field varies dramatically in this time interval, we estimate the magnetic curvature radius $\left(R_{c}\right.$; Figure $\left.2 \mathrm{~h}\right)$, defined by $\frac{\mathbf{R}_{\mathbf{c}}}{R_{c}^{2}}=\left(\frac{\mathbf{B}}{B} \cdot \nabla\right) \frac{\mathbf{B}}{B}$. It starts to decrease from 23:41:54.10 UT and reaches to its minimum $(\sim 66 \mathrm{~km})$ inside the depressed magnetic region. When $R_{c}$ is on the order of the gyroradius of a particle, the particle pitch angle scattering should occur (Büchner \& Zelenyi, 1989). Following Lavraud et al. (2016) and Zhang et al. (2016), we define an adiabatic parameter $\kappa^{2}=R_{c} / \rho_{e}$, and $\kappa^{2}$ values for four different electron energies are shown in Figure 2i. From 23:41:54.10 UT, the $\kappa^{2}$ values approach 25 (the dash line), suggesting that electron curvature scattering occurs, and inside the magnetic minimum region, the $\kappa^{2}$ values are well below 10 (the dotted line), implying that the electron orbits are chaotic as predicted by theory (Büchner \& Zelenyi, 1989). This means that the electron magnetic moment is not well conserved as an adiabatic invariant. Taking the maximum magnetic field strength of this interval $(\sim 16.2 \mathrm{nT})$ as a reference, the expected pitch angle of an adiabatic electron population should always stay above/below the two white dashed lines in Figure 2j. However, there are some electrons identified between the two dashed lines as a result of electron pitch angle scattering when $\kappa^{2}$ values are low enough from 23:41:54.10 UT, and the electron pitch angle mixing is more evident inside the magnetic minimum as expected. We estimate the density of scattered electrons with pitch angles between the two dashed lines and present it with a blue line $\left(N_{e^{\prime}}\right)$ in Figure 2c. It is found that nearly all electrons have been scattered in the magnetic minimum region.

The vectors of the magnetic field and electron bulk velocity for the four spacecraft in a three-dimensional view are plotted in Figures $2 \mathrm{k}$ and 2l, revealing large electron flows in the opposite direction at the two edges of the magnetic minimum. The similarity of the magnetic field and electron flow structures, although they are crossed at slightly different times by the respective spacecraft, suggests that the spatial scale of the observed structure is larger than MMS separations. The formation of this magnetic minimum is currently not clear.

Considering that the regular 30-ms electron measurement cannot well resolve the electron behaviors at the edges of the magnetic minimum, whose duration is about $0.1 \mathrm{~s}$, we use higher-resolution 7.5-ms electron data (Rager et al., 2018) to investigate the electron behaviors around the magnetic minimum. The thin electron-scale boundaries (Figure 3a) separate two different electron populations inside/outside the magnetic minimum. The electrons inside the magnetic minimum are more isotropic due to the strong mag- 


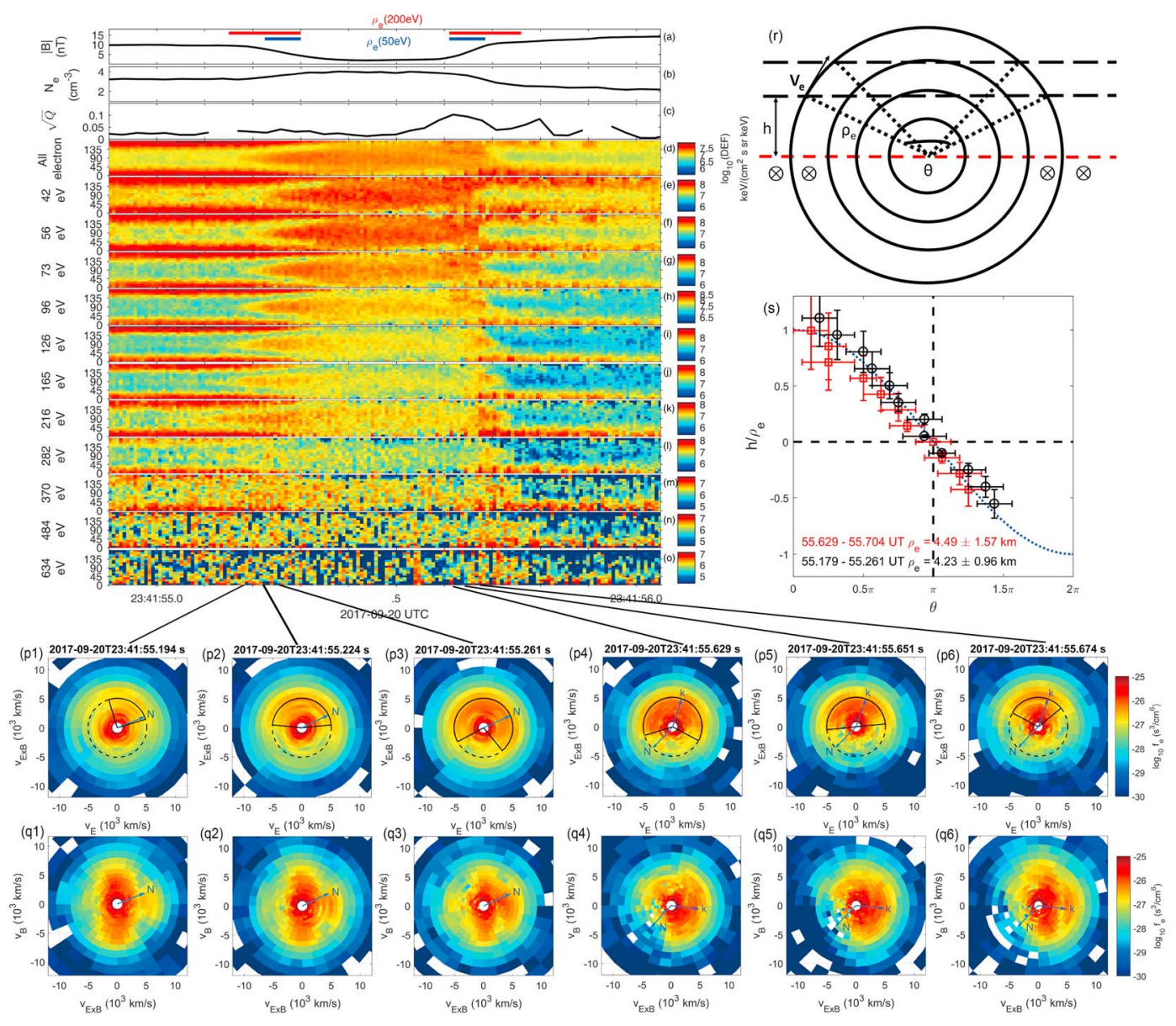

Figure 3. Electron distributions around the magnetic minima. (a) Magnetic field magnitude. (b) Electron density. (c) Agyrotropy measure $(\sqrt{Q})$. (d) Electron pitch angle distributions of all energies. (e-o) Electron pitch angle distributions of energy channels from 42 to $634 \mathrm{eV}$. (p1-p6) Six selected electron distributions in the perpendicular plane $\left(\mathbf{v}_{\mathbf{E}}-\mathbf{v}_{\mathbf{E} \times \mathbf{B}}\right)$. (q1-q6) The electron distributions in the plane $\left(\mathbf{v}_{\mathbf{E} \times \mathbf{B}}-\mathbf{v}_{\mathbf{B}}\right)$. The vectors "N" and "k" denote the projected normal direction and the propagation direction of upper hybrid waves discussed later. ( $\mathrm{r}$ ) A cartoon of the electron finite gyroradius effect under the uniform magnetic field. (s) The relation between the opening angle of the agyrotropic electrons $(\theta)$ and the distance to the boundary $(\mathrm{h})$.

netic curvature scattering, while the electrons are distributed more into the field-aligned direction outside (Figure 3c). At these narrow magnetic boundaries, electrons near $90^{\circ}$ pitch angles extend from the depressed magnetic region, and this tendency is much clearer for relatively high-energy electrons (Figures $3 \mathrm{e}-3 \mathrm{k}$ ). This can be explained by the electron finite gyroradius effect (Figure 3r), which shows how electrons inside the magnetic minimum can cross the magnetic boundary due to gyromotion, and this feature is more significant for higher-energy electrons with larger gyroradius.

These electrons at the thin magnetic boundary will form agyrotropic distributions perpendicular to the magnetic field according to the finite gyroradius effect. Crescent-shaped electron distributions can also be generated if these agyrotropic electrons are well separated from the core distributions. Figures 3p1-3p3 and 3p4-3p6 present such distributions at the two boundaries of the magnetic minimum in the perpendicular plane $\left(\mathbf{v}_{\mathbf{E}_{\perp}}-\mathbf{v}_{\mathbf{E} \times \mathbf{B}}\right)$ with the projection of $\mathbf{N}$ direction, and the agyrotropic electrons are mainly observed near $90^{\circ}$ pitch angles as expected (Figures 3q1-3q6). Basically, the angular range (or the opening angle) of the agyrotropic electron distributions in the perpendicular plane decreases and their phase space density becomes smaller as these electrons go further from the magnetic boundary. In addition, the agyrotropic electron distributions are roughly oriented in the opposite directions at the two edges as a result of electron gyromotion from the projected $\mathbf{N}$ direction (Figures 3p1-3p6), consistent with electron flows shown in Figure 2. Similar agyrotropic electron distributions have been revealed by all MMS spacecraft. The measure of electron agyrotropy, $\sqrt{Q}$ (Swisdak, 2016) in Figure 3c, remains small at the magnetospheric boundary, 

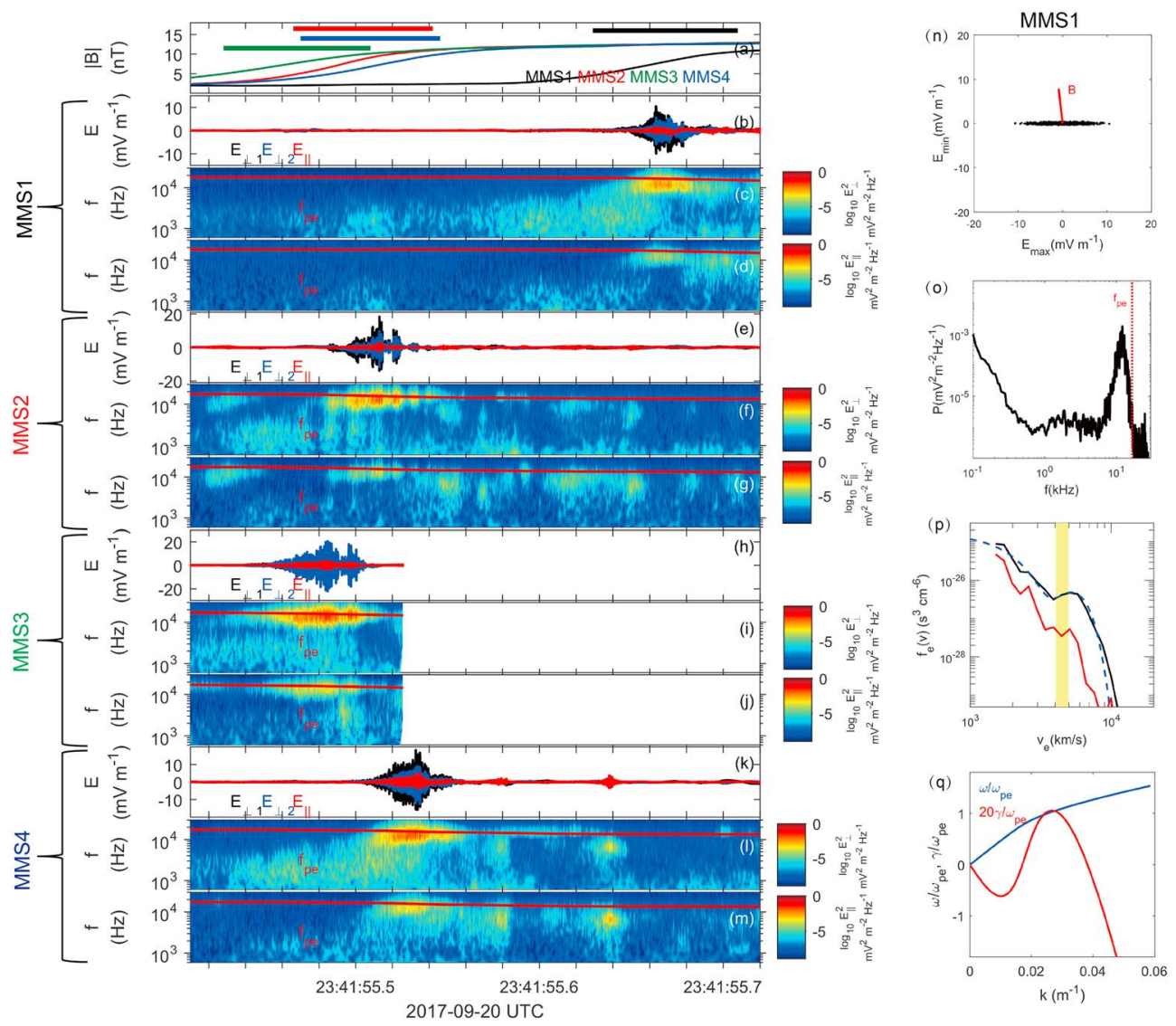

Figure 4. Upper hybrid waves associated with electron crescents. (a) Magnetic field magnitude of all MMS spacecraft and four color bars denote the time interval of the agyrotropic electron distributions. (b-d) MMS1 observations: electric field in field-aligned coordinate, spectral power density of the perpendicular electric field, and the parallel electric field. (e-g), (h-j), and (k-m): The same format for MMS2, MMS3, and MMS4. (n) Hodograms of $E_{\max }$ versus $E_{\min }$ of MMS1, and the red line is $\mathbf{B}$ direction. (o) Power spectrum of the electric field (the red dashed line is $f_{\mathrm{pe}}$ ). (p) One-dimensional electron distributions along $\mathbf{k}$ (black) and $\mathbf{- k}$ (red) during the electron crescent interval. The blue dashed line is a two-Maxwellian fit to the black curve, and the yellow-shaded region indicates the domain of electron speeds trapped by the wave potential. (q) The wave frequency $(\omega)$ and the linear growth rate $(\gamma)$ predicted from the fitted distribution. MMS = Magnetospheric Multiscale.

which can be attributed to the fact that the agyrotropic electron distributions cannot significantly contribute to the pressure tensor of the total distribution (Norgren et al., 2016).

Taking electrons of 5,000 km/s as a reference, the opening angle of the agyrotropic electrons indicated by the black lines is illustrated (Figures 3p1-3p6). Electrons with a particular velocity and gyroradius $\left(V_{e}\right.$ and $\left.\rho_{e}\right)$ can only be detected inside a limited angle range $\left(\theta\right.$, the opening angle) at the distance $h\left(h<\rho_{e}\right)$ from the boundary (Figure 3r). The relation between $h$ and $\theta$ can be written as $h=\rho_{e} \cos \left(\frac{\theta}{2}\right)$ from geometry, and it has been further checked by the 7.5-ms electron data at these two boundaries (black and red symbols in Figure $3 \mathrm{~s}$ ). The errors of the normalized distance come from the nonuniform magnetic field, while the horizontal bars indicate uncertainties in determining the open angles of the agyrotropic electron distributions. In general, good agreement is found between observations and predictions (the dotted green line) of the finite gyroradius effect.

These agyrotropic electron distributions are not in equilibrium state, if crescent distributions are presented (i.e., Figures 3p6 and 3q6). The existence of a positive slope in electron velocity distributions $\left(+\frac{\partial f}{\partial \nu}\right)$ is unstable for plasma waves (e.g., Graham et al., 2017b; Landau, 1946). At the magnetosheath boundary of the magnetic minimum, all MMS spacecraft observe wave activities accompanied with electron crescents indicated by different color bars (Figure $4 \mathrm{a}$ ). Figures $4 \mathrm{~b}-4 \mathrm{~d}$ show the waveform of the high-frequency $\mathbf{E}$ perpendicular and parallel to $\mathbf{B}$ of MMS1 and the related spectrogram of $\mathbf{E}$. The wave electric field is almost in the perpendicular direction with a peak wave amplitude at $\sim 12 \mathrm{mV} / \mathrm{m}$ and the wave frequency close to the 
plasma frequency $\left(f_{\mathrm{pe}}=16.6 \mathrm{kHz}\right)$. These waves then are identified as UH waves (Graham et al., 2017b). Similar wave features are observed by the other MMS spacecraft.

Properties of the UH waves are further investigated. Based on minimum variance analysis of the wave electric field, we can find the electric fields in the maximum $\left(E_{\max }\right)$ and intermediate and minimum $\left(E_{\min }\right)$ variance directions and present the hodogram of the waveform $\left(E_{\max }\right.$ and $\left.E_{\min }\right)$ in Figure 4 n. The ratio among the maximum, intermediate, and minimum eigenvalues is about 500:6:1, suggesting that the waveform is almost one dimensional and linearly polarized. Due to the electrostatic nature of the UH waves, the wave $\mathbf{k}$ vector is aligned with maximum variance direction, pointing to the unstable electron crescent (Figures 3p4-3p6). The propagation direction $\mathbf{k} / k$ is determined to be $[-0.57,-0.36,-0.74]$ (GSM), which is about $96^{\circ}$ from $\mathbf{B}$. One-dimensional electron distributions along $\mathbf{k}$ and $-\mathbf{k}$ are shown in Figure $4 \mathrm{p}$. The crescent, with a positive slope in electron velocity distributions, is the only apparent energy source of the $\mathrm{UH}$ waves, and the estimated energy density of the crescent $\left(n_{c} m_{e} v_{c}^{2} / 2 \approx 1 . \times 10^{-12} \mathrm{~J} / \mathrm{m}^{3}\right.$, and the parameters are estimated from the fitting below) is orders of magnitude larger than the wave energy density $\left(\epsilon_{0}|\mathbf{E}|^{2} / 2 \approx 7 . \times 10^{-16} \mathrm{~J} / \mathrm{m}^{3}\right)$.

By fitting the observed electron distribution along $\mathbf{k}$ into two Maxwellian distributions (the dotted blue line in Figure 4p) and assuming that the electrons are weakly magnetized as wave frequency is much larger than the electron gyrofrequency $\left(\Omega_{e}\right)$, we solve the linear dispersion equation in waves in homogeneous, anisotropic, multicomponent plasmas (Rönnmark, 1982) and show the predicted wave mode in Figure 4q. The mode has a maximum linear growth range of $\gamma=0.05 \omega_{\mathrm{pe}} \approx 3 \sim 6 \Omega_{e}$ around $\omega=\omega_{\mathrm{pe}}$, allowing waves to grow fast. At the peak $\gamma$, the wavelength $\lambda \approx 240 \mathrm{~m}$ or $10 \lambda_{D}$ ( $\lambda_{D}$ is the Debye length), which is much smaller than the electron gyroradius. The wave speed $\left(v_{\mathrm{ph}}\right)$ then is estimated at $4,500 \mathrm{~km} / \mathrm{s}$. From the estimated $v_{\mathrm{ph}}$ and the observed $E_{\max }$, the wave potential $(\Phi)$ is about $0.6 \mathrm{~V}$. Electrons with speed $v_{T}=v_{\mathrm{ph}} \pm \sqrt{2 q_{e} \Phi / m_{e}} \approx$ $[4.5 \pm 0.5] \times 10^{3} \mathrm{~km} / \mathrm{s}$ can be trapped by waves (shaded by the yellow region in Figure $4 \mathrm{p}$ ), which overlaps most of the positive slope in the electron distribution. Thus, the positive slope can be relaxed by wave-particle interactions, as suggested by Graham et al. (2017b).

\section{Discussion and Summary}

We presented MMS observations at Earth's flank magnetopause on 20 September 2017. During the magnetopause crossing, highly curved magnetic field lines with a magnetic minimum are identified, whose curvature radius is comparable to the electron gyroradius. Thus, the electron pitch angle mixing occurs due to the curvature scattering, and the scattered electron density inside the magnetic minimum is close to the total electron density. Agyrotropic electron distributions are then found at the two thin electron-scale boundaries of the magnetic minimum due to electron gyromotion. With the help of 7.5-ms MMS data, a quantitative analysis of the opening angle of these agyrotropic electron distributions was performed, indicating that the angular range of the agyrotropic electron distributions decreases with distance. We note that this opening angle variation also depends on the electron energy, but we only investigate the opening angle of the agyrotropic electrons at its typical energy ( $\sim 0 \mathrm{eV}$ or $5,000 \mathrm{~km} / \mathrm{s})$ as presented in Figure 4q. The reasons are as follows: (1) we emphasize the electron gyromotion to create the agyrotropic electron distributions, but the electron drift motion is ignored when estimating the opening angle, which is around 1,000 $\mathrm{km} / \mathrm{s}$ at the two thin magnetic boundaries. To make this assumption more reliable, we should select electrons with a speed much larger than the drift speed. (2) Due to the wave-particle interactions, some electrons around the UH wave speed could be transported from higher energies when relaxing the positive slope in the electron velocity distributions. These wave-particle interactions limit the electron energy selection to analyze the opening angle of the agyrotropic electron distributions.

Previous studies have revealed the agyrotropic electron crescent distributions in the EDR or near-EDR region during magnetic reconnection, and therefore, they are taken as one of the observational signatures of an EDR and near-EDR event (e.g., Fuselier et al., 2017; Webster et al., 2018). However, signatures of fast reconnection are lacking in this event, though the magnetic shear of the overall current sheet is approximately $140^{\circ}$ : (1) MMS do not observe large ion jets in the maximum magnetic shear direction $\left(v_{i, L}\right),(2)$ electrons still follow the $\mathbf{E} \times \mathbf{B}$ drift motion, (3) the lacking of positive $\mathbf{J} \cdot E^{\prime}$ (Figure $2 \mathrm{~g}$ ) indicates that the conversion from magnetic energy to particle energy is weak, (4) the agyrotropic electrons cannot be well separated from the core distribution (Figures $3 p 3$ and 3p4) until they gyrate further away from the magnetic boundary (Figures 3p1 and 3p6). The typical energy of crescent electrons is about $70 \mathrm{eV}$ (Figure 4q), which 
Acknowledgments

We thank the entire MMS team for providing high-quality data. MMS data can be accessed from MMS Science Data Center (https://lasp.colorado.edu/mms/ sdc/public/). This work was supported by the National Natural Science Foundation of China (grants 41474145 , 41504114, 41574159, and 41731070), Chinese Academy of Sciences (QYZDJ-SSW-JSC028 and XDA15052500), and the Specialized Research Fund for State Key Laboratories of China. is similar to the background sheath electrons (Figure $1 \mathrm{j}$ ). This indicates that the electron energization in this event is not obvious, which is significantly different from the process in the reconnection EDR (e.g., Argall et al., 2018; Burch et al., 2016; Chen et al., 2016). (5) The agyrotropic electrons here are mainly distributed around the perpendicular plane. These agyrotropic distributions are explained by electron gyromotion at thin electron-scale boundaries and could be different from the EDR cases, where the transition from perpendicular crescents into parallel crescents has been observed owing to the parallel electric field and/or the electron inertia (Burch et al., 2016; Egedal et al., 2018). Based on the observations above, we conclude that reconnection here is not active, and a similar electron-scale current sheet without bursty reconnection signatures has been reported in the magnetotail (Wang et al., 2018). Hence, the electron crescents can form at the magnetopause more frequently, where reconnection is not necessary, and cover a large local time range to the flank region.

The average separation of the four MMS spacecraft is about $20 \mathrm{~km}$, which is larger than the electron gyroradius at the observed crescents $(\sim 4.5 \mathrm{~km})$ and the wavelength of UH waves $(\sim 240 \mathrm{~m})$, but all MMS spacecraft have observed similar magnetic field structures, electron crescent distributions, and UH waves. Though the wave amplitude recorded by other three MMS spacecraft ( $>20 \mathrm{mV} / \mathrm{m})$ is larger than that at MMS1, the wave propagation directions among these spacecraft, with a difference less than $20^{\circ}$, are all aligned with the electron crescents. This indicates that UH waves can be excited along the thin magnetic boundary within a length much longer than MMS separations. The estimated wave potential $(0.6 \mathrm{~V}$ or higher) can relax the positive slope of electron distributions and thus thermalize electrons. Actually, these UH waves and other high-frequency electrostatic waves are frequently found at the magnetopause (Graham et al., 2018).

\section{References}

Argall, M. R., Paulson, K., Alm, L., Rager, A., Dorelli, J., Shuster, J., et al. (2018). Electron dynamics within the electron diffusion region of asymmetric reconnection. Journal of Geophysical Research: Space Physics, 123, 146-162. https://doi.org/10.1002/2017JA024524

Bessho, N., Chen, L.-J., Hesse, M., \& Wang, S. (2017). The effect of reconnection electric field on crescent and U-shaped distribution functions in asymmetric reconnection with no guide field. Physics, Plasmas, 24, 72903. https://doi.org/10.1063/1.4989737

Büchner, J., \& Zelenyi, L. M. (1989). Regular and chaotic charged particle motion in magnetotail-like field reversals: 1 . Basic theory of trapped motion. Journal of Geophysical Research, 94(A9), 11,821-11,842. https://doi.org/10.1029/JA094iA09p11821

Burch, J. L., \& Phan, T. D. (2016). Magnetic reconnection at the dayside magnetopause: Advances with MMS. Geophysical Research Letters, 43, 8327-8338. https://doi.org/10.1002/2016GL069787

Burch, J. L., Torbert, R. B., Phan, T. D., Chen, L.-J., Moore, T. E., Ergun, R. E., et al. (2016). Electron-scale measurements of magnetic reconnection in space. Science, 352(6290), aaf2939. https://doi.org/10.1126/science.aaf2939

Burch, J. L., Webster, J. M., Genestreti, K. J., Torbert, R. B., Giles, B. L., Fuselier, S. A., et al. (2018). Wave phenomena and beam-plasma interactions at the magnetopause reconnection region. Journal of Geophysical Research: Space Physics, 123, 1118-1133. https://doi.org/10.1002/2017JA024789

Chen, L.-J., Hesse, M., Wang, S., Gershman, D., Ergun, R., Pollock, C., et al. (2016). Electron energization and mixing observed by MMS in the vicinity of an electron diffusion region during magnetopause reconnection. Geophysical Research Letters, 43, 6036-6043. https://doi.org/10.1002/2016GL069215

Egedal, J., Le, A., Daughton, W., Wetherton, B., Cassak, P., Burch, J., et al. (2018). Spacecraft observations of oblique electron beams breaking the frozen-in law during asymmetric reconnection. Physical Review Letters, 120, 55101.

Egedal, J., Le, A., Daughton, W., Wetherton, B., Cassak, P. A., Chen, L.-J., et al. (2016). Spacecraft observations and analytic theory of crescent-shaped electron distributions in asymmetric magnetic reconnection. Physical Review Letters, 117, 185101. https://doi.org/10.1103/PhysRevLett.117.185101

Ergun, R. E., Tucker, S., Westfall, J., Goodrich, K. A., Malaspina, D. M., Summers, D., et al. (2014). The axial double probe and fields signal processing for the MMS mission. Space Science Reviews, 199, 167-188. https://doi.org/10.1007/s11214-014-0115-x

Fuselier, S. A., Vines, S. K., Burch, J. L., Petrinec, S. M., Trattner, K. J., Cassak, P. A., et al. (2017). Large-scale characteristics of reconnection diffusion regions and associated magnetopause crossings observed by MMS. Journal of Geophysical Research: Space Physics, 122, 5466-5486. https://doi.org/10.1002/2017JA024024

Graham, D. B., Khotyaintsev, Y. V., Norgren, C., Vaivads, A., André, M., \& Toledo-Redondo, S. (2017a). Lower hybrid waves in the ion diffusion and magnetospheric inflow regions. Journal of Geophysical Research: Space Physics, 122, 517-533. https://doi.org/10.1002/2016JA023572

Graham, D. B., Khotyaintsev, Y. V., Norgren, C., Vaivads, A.,André, M., Toledo-Redondo, S., et al. (2017b). Instability of agyrotropic electron beams near the electron diffusion region. Physical Review Letters, 119, 25101. https://doi.org/10.1103/PhysRevLett.119.025101

Graham, D. B., Vaivads, A., Khotyaintsev, Y. V., Andre, M., Le Contel, O., Malaspina, D. M., et al. (2018). Large-amplitude high-frequency waves at Earth's magnetopause. Journal of Geophysical Research: Space Physics, 123, 2630-2657. https://doi.org/10.1002/2017JA025034

Hesse, M., Aunai, N., Sibeck, D., \& Birn, J. (2014). On the electron diffusion region in planar, asymmetric systems. Geophysical Research Letters, 41, 8673-8680. https://doi.org/10.1002/2014GL061586

Khotyaintsev, Y. V., Graham, D. B., Norgren, C., Eriksson, E., Li, W., Johlander, A., et al. (2016). Electron jet of asymmetric reconnection. Geophysical Research Letters, 43, 5571-5580. https://doi.org/10.1002/2016GL069064

Landau, L. D. (1946). On the vibration of the electronic plasma. Journal of Physics, 10, 25-34. Moscow.

Lapenta, G., Berchem, J., Zhou, M., Walker, R. J., El-Alaoui, M., Goldstein, M. L., et al. (2017). On the origin of the crescent-shaped distributions observed by MMS at the magnetopause. Journal of Geophysical Research: Space Physics, 122, 2024-2039. https://doi.org/10.1002/2016JA023290 
Lavraud, B., Zhang, Y. C., Vernisse, Y., Gershman, D. J., Dorelli, J., Cassak, P. A., et al. (2016). Currents and associated electron scattering and bouncing near the diffusion region at Earth's magnetopause. Geophysical Research Letters, 43, 3042-3050. https://doi.org/10.1002/2016GL068359

Lindqvist, P.-A., Olsson, G., Torbert, R. B., King, B., Granoff, M., Rau, D., et al. (2014). The spin-plane double probe electric field instrument for MMS. Space Science Reviews, 199, 137-165. https://doi.org/10.1007/s11214-014-0116-9

Norgren, C., Graham, D. B., Khotyaintsev, Y. V., André, M., Vaivads, A., Chen, L.-J., et al. (2016). Finite gyroradius effects in the electron outflow of asymmetric magnetic reconnection. Geophysical Research Letters, 43, 6724-6733. https://doi.org/10.1002/2016GL069205

Phan, T. D., Eastwood, J. P., Shay, M. A., Drake, J. F., Sonnerup, B. U. Ö., Fujimoto, M., et al. (2018). Electron magnetic reconnection without ioncoupling in Earth's turbulent magnetosheath. Nature, 557(7704), 202-206. https://doi.org/10.1038/s41586-018-0091-5

Phan, T. D., Shay, M. A., Haggerty, C. C., Gosling, J. T., Eastwood, J. P., Fujimoto, M., et al. (2016). Ion Larmor radius effects near a reconnection X line at the magnetopause: THEMIS observations and simulation comparison. Geophysical Research Letters, 43, 8844-8852. https://doi.org/10.1002/2016GL070224

Pollock, C., Moore, T., Jacques, A., Burch, J., Gliese, U., Saito, Y., et al. (2016). Fast plasma investigation for magnetospheric multiscale. Space Science Reviews, 199, 331-406. https://doi.org/10.1007/s11214-016-0245-4

Rager, A. C., Dorelli, J. C., Gershman, D. J., Uritsky, V., Avanov, L. A., Torbert, R. B., et al. (2018). Electron crescent distributions as a manifestation of diamagnetic drift in an electron-scale current sheet: Magnetospheric Multiscale observations using new 7.5 ms Fast Plasma Investigation moments. Geophysical Research Letters, 45, 578-584. https://doi.org/10.1002/2017GL076260

Rönnmark, K. (1982). WHAMP: Waves in homogeneous, anisotropic, multicomponent plasmas (Tech. Rep.) Kiruna, Sweden: Kiruna Geophys. Inst.

Russell, C. T., Anderson, B. J., Baumjohann, W., Bromund, K. R., Dearborn, D., Fischer, D., et al. (2014). The magnetospheric multiscale magnetometers. Space Science Reviews, 199, 189-256. https://doi.org/10.1007/s11214-014-0057-3

Shay, M. A., Phan, T. D., Haggerty, C. C., Fujimoto, M., Drake, J. F., Malakit, K., et al. (2016). Kinetic signatures of the region surrounding the X line in asymmetric (magnetopause) reconnection. Geophysical Research Letters, 43, 4145-4154. https://doi.org/10.1002/2016GL069034

Swisdak, M. (2016). Quantifying gyrotropy in magnetic reconnection. Geophysical Research Letters, 43, 43-49. https://doi.org/10.1002/2015GL066980

Wang, S., Chen, L.-J., Hesse, M., Bessho, N., Gershman, D. J., Dorelli, J., et al. (2016). Two-scale ion meandering caused by the polarization electric field during asymmetric reconnection. Geophysical Research Letters, 43, 7831-7839. https://doi.org/10.1002/2016GL069842

Wang, R., Lu, Q., Nakamura, R., Baumjohann, W., Huang, C., Russell, C. T., et al. (2018). An electron-scale current sheet without bursty reconnection signatures observed in the near-Earth tail. Geophysical Research Letters, 45, 4542-4549. https://doi.org/10.1002/2017GL076330

Webster, J. M., Burch, J. L., Reiff, P. H., Daou, A. G., Genestreti, K. J., Graham, D. B., et al. (2018). Magnetospheric Multiscale dayside reconnection electron diffusion region events. Journal of Geophysical Research: Space Physics, 123, 4858-4878. https://doi.org/10.1029/2018JA025245

Zhang, Y. C., Shen, C., Marchaudon, A., Rong, Z. J., Lavraud, B., Fazakerley, A., et al. (2016). First in situ evidence of electron pitch angle scattering due to magnetic field line curvature in the Ion diffusion region. Journal of Geophysical Research: Space Physics, 121, 4103-4110. https://doi.org/10.1002/2016JA022409 